கிழக்கிலங்கை வேடர்

வழிபாட்டுச்

சடங்குகளில்

தொல்சமய

அடிப்படைகள்

த. விவானந்தராசா அ, *

அ புதுச்சேரி மொழியியல் பண்பாட்டு ஆராய்ச்சி நிறுவனம், புதுச்சேரி-605008, இந்தியா.

\title{
Archaeological Fundamentals in Eastern Vader Worship Rituals
}

\author{
T. Vivanantaraca ${ }^{a}$, \\ a Puducherry Institute of Linguistics and Culture, Puducherry- 605008, India
}

* Corresponding Author: vivanantharsa@gmail.com

Received: 04-01-2021

Revised: 13-04-2021

Accepted: 15-04-2021

Published: 30-04-2021

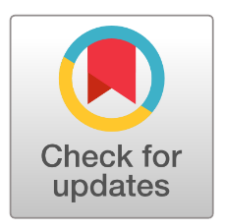

\begin{abstract}
Hunter society is considered as an indigenous population in Sri Lanka and is one of the fewest communities in number. The Hunter community, which has to live in the east with large communities of Tamils, Sinhalese and Tamil speaking Muslims, is losing its cultural identity. However, it continues to study unique cultural performances, as it is not completely free from the traditional tribe slife. The rituals of worship are also important in these performing symmetry, and the article symbolizes the archaeological foundations found in these rituals. These rituals also focus on the methods of stabilizing the hunter sociocultural life.
\end{abstract}

Keywords: Biology, Performance, Antiquity, Anonymity.

\section{முன்னுரை}

உலகம் முழுவதுமே வர்க்கச் சமூகம் உருவாகியதன் உடன் விளைவாகத் தொல்சமயம் சிதைந்து, நிறுவனச் சமயம் உருவானது. ஆயினும் உலகில் தொல்சமயத்தின் எச்சங்கள் தொடர்ந்து நிலவி வருகின்றன (Sivasubramaniyan, 2018: 16). கிழக்கிலங்கையில் பூர்வீகக் குடியாகக் கருதப்படும் வேடர் சமூகத்தின் வழிபாட்டுச் சடங்குகளிலும் தொல்சமயத்தின் தொடர்ச்சியை அடையாளங் காணமுடிகிறது.

இலங்கையில் வேடர் சமூகத்தினர் பரவலாக வாழும் ஒரு சில பகுதிகளுள் கிழக்கு மாகாணமும் ஒன்று. மட்டக்களப்பு, திருகோணமலை, அம்பாரை ஆகிய மாவட்டங்களை உள்ளடக்கிய கிழக்குமாகாணமே கிழக்கிலங்கை எனவும் அழைக்கப்படுகின்றது. கிழக்கிலங்கையில் தமிழர், சிங்களவர், தமிழ்பேசும் முஸ்லிம் பெரும் சமூகத்தினராக வாழ்ந்து வருகின்றனர். இ இந்தப் பெருஞ்சமூகங்களுடன் கலப்புற்று வாழவேண்டிய நிலைமை வேடர்கள் வரலாற்றில் தொடர்ந்து நிகழ்ந்து வந்துள்ளது. இது வேடர்தம் பண்பாட்டு அடையாளத்தை இழந்து வருவதன் வரலாறாகவும் இருந்து வருகிறது.

காலனிய ஆட்சியாளர்கள் முதல் நாகரிகமயமாக்கல் படிமுறைக்கும், பின்பு தமிழ்த்தேசியத்துடனான ஒருங்கிணைப்புக்கும் பலியாகியுள்ள உணர்வே வேடர் தமது அடையாளத்தை மீள முன்வைப்பதை விளக்கும் (Thangarasa, 1994). இன முரண்பாட்டால் நாட்டில் நீண்டகாலமாக நிலவிய ஆயுத மோதல்கள் முதல் எல்லாமே சுமுகமாக முடிவுற்றதாகச் சொல்லப்படும் இன்றைய சூழல்வரை வேடர், தமது இயல்பு வாழ்க்கைக்குத் திரும்ப மமடியவில்லை. இனக்குழு வாழ்வியலிலிருந்து 
பெயர்க்கப்பட்டதன் விளைவுகள் பாரதூரமானதாக அமைந்திருப்பதாக மூத்த தலைமுறையினர் பலரும் முணுமுணுக்கின்றனர். எனவே அரசாங்கத்திடமிருந்தோ வேறு சமூகங்களிடமிருந்தோ எந்தக் குறுக்கீடுமன்றி தமது பாரம்பரிய வாழ்க்கை முறையைத் தொடரவேண்டுமென வேடர்களின் ஒரு பிரிவினர் கோருகின்றனர். மறுபுறம் பிற சமூகங்களுடன் கலப்புற்று வாழ்ந்துவரும் நீண்டகால வாழ்க்கைத் தொடர்ச்சியில் யதார்த்த நிலவரங்களை ஏ ஏற்றுப் பயணித்தாக வேண்டிய நிர்ப்பத்தங்களையும் சிலர் எடுத்துரைக்கின்றனர்.

மொத்தத்தில் பெரும்பான்மைப் பண்பாடு எங்கும் பொதுத் தன்மையாக்கப்படுகிறது. தமது இருப்பிற்கும் அடையாளத்திற்குமென வேடர்கள் பயின்றுவந்த பண்பாட்டு நிகழ்த்துகைகள் மறைந்தழிந்துபோகின்றன. குறிப்பாக பொருளாதார உற்பத்தி முறையில் நிகழ்ந்துள்ள மாற்றங்கள் அவர்களது வாழ்வியல் மாற்றங்களை நிச்சயப்படுத்தியுள்ளன. எவ்வாறாயினும் வேடர் சமூகம் தமது தனித்துவமான இனக்குழு வாழ்வினை முற்றாக இழந்துவிடவில்லை.

அவற்றின் தக்கவைப்பிற்கான முயற்சிகளிலிருந்து ஒதுங்கிவிடவுமில்லை. மரபுமாறாத நிலையில் நம்பிக்கையின் அடிப்படையில் இவர்களால் நிகழ்த்துப்பட்டு வரும் வழிபாட்டுச் சடங்குகள் தக்க எடுத்துக்காட்டுகளாகின்றன.

இக்கட்டுரை, கிழக்கிலங்கையின் பூர்வீகக் குடியாக இருக்கும் வேடர்களது வழிபாட்டுச் சடங்கில் தொல்சமயத்தின் அடிப்படைகளை அடையாளங்காட்ட முயற்சிக்கிறது. மறுபுறம் இச்சடங்குகள் வேடர்களது சமூக பண்பாட்டு வாழ்வியலை வலுப்படுத்தும் முறைகளையும் ஆராய்கிறது.

இந்தக் கட்டுரைக்கான ஆய்வுத் தரவாக கிழக்கிலங்கையில் வேடர்கள் செறிந்து வாழும் குஞ்சன்கல்குளம், பாற்சேனை, சூரநகர், களுவன்கேணி ஆகிய கிராமங்களில் நிகழும் வழிபாட்டுச் சடங்குகள் அமைகின்றன. இனவரைவியல் அணுகுமுறையில் தரவுகள் சேகரிக்கப்பட்டுள்ளன. இவ்வாய்வுக் கட்டுரைக்கான கோட்பாட்டு அணுகுமுறையானது தமிழ்மரபில் தொல்சமயம் குறித்து விரிவாக விளக்கும் ஆ. சிவசுப்பிரமணியன் அவர்களது 'மந்திரமும் சடங்கும்' எனும் நூல் வழியும் பக்தவத்சல பாரதி அவர்களது ‘மானிடவியல் கோட்பாடுகள்' நூல் வழியும் பெறப்பட்டுள்ளது.

\section{தொல்சமயம்}

தனக்கென வரையறுக்கப்பட்ட விதிமுறைகளையும், கோட்பாடுகளையும் தத்துவங்களையும், அமைப்புக்களையும் கொண்டுள்ள சமயங்களை நிறுவனச் சமயம் என்று ஆய்வாளர்கள் குறிப்பிடுவர். இவ்வரையறைக்கு வராது இருக்கும் சமயங்களும் உண்டு. தொல்சமயம் (Pசiஅவைiஎந சநடபைழைn), நாட்டார் சமயம் (Folk religion) என்பன அவற்றுள் அடங்கும் (Sivasubramaniyan, 2017: 184).

தொல்சமயம் என்று பொதுவாகக் குறிப்பிட்டாலும், ஒரே வகையான சமய நெறியாக இதைக் குறிப்பிட்டுவிட முடியாது. இதில் பல படிநிலைகள் உண்டு. மானிடவியலாளர் கருத்துப்படி தொல்சமயம், ஆவியியம், குலக்குறியியம், இயற்கைப் பொருள் வழிபாடு, வெறியாட்டு முதலான முக்கிய கூறுகளைக் கொண்டது. தொல்சமய வழிபாடுகளில் இறந்தோர் மற்றும் மூத்தோர் வழிபாடு, உயிர்ப்பலி, புனித - புனிதமற்றவை என்று இன்று நாம் பாகுபடுத்துவது போலன்றி இறைச்சிகள் ஆகியனவற்றை படையல்களாகப் படைத்து வழிபடல், இவை அனைத்தையும் இணைக்கும் சரடாக ஆவி என்ற கண்ணுக்குப் புலனாகாத ஒரு பொருள் குறித்த நம்பிக்கை முதலானவை அடிப்படை மரபுகளாகக் காணப்படுகின்றன (Sivasubramaniyan, 2017: 184)

மனித ஆற்றலுக்கு மேற்பட்ட ஓர் ஆற்றல் உள்ளது என்ற நம்பிக்கை மற்றும் அச்சத்தின் அடிப்படையில் உருவான தொல்சமயம், ஆகம விதிமுறைகளுக்குட்பட்ட சடங்குகள், புராணக் கோட்பாடுகள் ஆகியவற்றின் தாக்குதலால் சிதைந்துபோயுள்ளது. இச்சிதைவின்மேல் வேத நெறிகளை உள்வாங்கிக்கொண்ட சமய நெறிகள் தம்மை நிலைநிறுத்திக் கொண்டன. என்றாலும் தொல்சமயத்தின் மரபுகளை முற்றாக அழிக்கவோ மாற்றவோ முடியவில்லை. மதமாற்றச் செயற்றிட்டங்கள் பரவலாக நிகழும் ூூழலிலும் அடித்தளமக்கள் வாழ்வில் தொல்சமயத்தின் எச்சங்கள் தொடர்ந்தவாறே இருக்கின்றன. கிழக்கிலங்கை வேடர் மரபிலும் இந்நிலைமையே தொடர்கிறது. 


\section{கிழக்கிலங்கை வேடர்கள் இனக்குழு அடையாளத்தால் பாதிக்கப்படுதல்}

கிழக்கிலங்கையில் ேேடர் சமூகத்தின் அடையாள மாற்றுருவாக்கத்தினை மூன்று கட்டங்களில் இனங்கண்டு கொள்ளமுடியும். 'காடுகளில் பயின்ற இனக்குழு வாழ்க்கையி லிருந்து மீட்கப்பட்டு’ குளங்களின் அருகே குடியமர்த்தப்பட்டது. இரண்டாவது, அவர்கள் மேலும் கரையை நோக்கி நகர்ந்து நாட்கூலிகளானமை மூன்றாவதும் இறுதியுமான கட்டம், இன முரண்பாட்டால் நீண்ட காலம் பாதிக்கப்பட்டு, அவர்களுக்கு அடிப்படை நிவாரணப் பொதியும் மறுக்கப்பட்டமை (Thangarasa, 1994: 50). போர் முடிவுற்றதாகச் சொல்லப்படும் அண்மைக் காலத்தில் வேடர்கள் எதிர்கொள்ளும் வாழ்வாதாரச் சவால்கள் உலகமயமாக்கல் சூழல் சுமத்தியிருக்கும் தொல்லைகள் என நான்காவது, ஐந்தாவது காரணங்களையும் அடுக்கிச் செல்லலாம். இவையாவும் வேடர்கள் இனரீதியான தனித்துவமான சமூகம் என்பதை அழித்தொழித்து வருகின்றன. எவ்வாறாயினும் வேடர் சமூகத்தின் பலர் தமது மரபார்ந்த வாழ்வை இன்னும் இழந்துவிடவில்லை தொடர்ந்து பயின்று வருகின்றனர்.

கிழக்கிலங்கையில் இ இற்கையுடன் நெருக்கம் கொண்ட இனக்குழு வாழ்வைத் தொடரும் கிராமங்களைக் காணமுடிகின்றது. முறுத்தானை, குஞ்சன்கல்குளம், கரடியன்குளம், பச்சநூல், பாட்டாளிபுரம் எனப் பல்கிராமங்கள் வேடர் கிராமங்களாகவே அறியப்படுகின்றன. இக்கிராமங்களில் நிகழ்த்தப்படும் வழிபாட்டுச் சடங்குகளை இங்கு கவனிக்கலாம்.

\section{வேடர் சமூக வழிபாட்டுச் சடங்குகள்: தொல்சமயக் கூறுகளும் அவை தரும் பண்பாட்டு அடையாளங்களும்}

இறந்த தமது மூதாதையரை வழிபடுவதென்பது வேடர் வழிபாட்டு மரபில் பிரதானமானதாக இருக்கிறது. உத்தியாக்கள்’ சடங்கு எனவும் சீயாக்களுக்கான சடங்கு’ எனவும் இதைக் குறிப்பிடுகின்றனர். இறந்த மூூதாதையர் தம்முடன் உடனிருப்பதாகவும் தம்மை வழி நடத்துவதாகவும் நம்பும் வேடர்கள் சடங்கில் எத்தனை தெய்வங்கள் இருந்தாலும் உத்தியாக்களே முதலில் வரும் (Shanmugam, 2018; Velayutham, 2019) என்கின்றனர். அதாவது தெய்வம். தெய்வாதியின் (தெய்வத்திற்கு சடங்கில் ஆடுபவர்) உடலில் வந்துறையும். இறந்த மூதாதையர் உறவினர் வேண்டுதலைக் கேட்டு, ஊரின் வேண்டுதலை ஏற்று தெய்வமாடுபவர் உடலில் வெளிப்பட்டு நிற்கும், சிக்கலைத் தீர்த்திடும் உபாயங்கள் உரைக்கும் என்கிறார் வேலாயுதம்.

வேடர் மரபில் குடிவழமை முக்கியமான ஒன்றாக உள்ளது. இதன்படி குடிக்கு குடி தெய்வங்களும் பலவாகக் காணப்படுகின்றன. மாறாத் தெய்வம், குடாட நீலி, தெவுத்தன், கப்பல் தெய்வம் எனப் பல. எதுவாயினும் எல்லாக்குடியிலும் பொதுவாக அமைவது உத்தியாக்கள் வழிபாடு ஆகும். எல்லாத் தெய்வங்களும், தெய்வங்களது ஆவிகளும் தெய்வாதியின் உடலில் தாவி அடையாளம் காட்டும். பந்தல்கள் (வழிபாட்டுக் கூடம்) அமைத்து பூக்கள், இலை குழைகள் காய்கள் முதலானவைகளையும் தமது மரபுத் தொழிலான வேட்டை, தேன் வெட்டுதல், சேனைப் பயிர் வைத்தல்) ஆயுதங்களான வில், அம்பு, ஈட்டி முதலானவைகளையும் வைத்து வழிபாடு தொடங்குகிறது.

தெய்வாதி, ‘கப்புகன்’ (பூசையை நடாத்துபவர் - சில இடங்களில் இவர் ‘வளக்கட்டியா’ எனவும் அழைக்கப்படுகிறார்) பூக்கள் பொறிக்கப்பட்ட துணிகளை இடுப்பில் துண்டுகளாகவும் மேற் சட்டைகளாகவும் அணிந்திருக்கின்றனர். சில தெய்வங்களுக்கு ஆடுவோர் தென்னங் குருத்தை பின்னி ஆடையாக அணிந்திருக்கின்றனர். கொட்டிசைக்கும் பாட்டுக்கும் உருக்கொண்டு தெய்வாதி ஆடுகிறார். ஈட்டி, வில் முதலியவற்றை எடுத்து ஆடுகிறார், ஆர்ப்பரித்து குரலொலிகளை எழுப்புகிறார்கள், பின்னர் கப்புகனிடம் கட்டும் சொல்கிறார். தெய்வாதி உடலில் உருக்கொண்டிருக்கும் உத்தியாக்கள் முதலில் சடங்கு செய்ய அனுமதியும் ஆசியும் வழங்குவர். (வேலாயுதம், குஞ்சன்கல்குளம்). இங்கு பாடப்படும் பாடல் முதல் உரையாடல்கள் யாவும் வேட மொழியில் அமைந்திருப்பதும் கவனிக்கத்தக்கது. உத்தியாக்களுக்கான பாடல் இவ்வாறமைகிறது. 
கண்டா பெடியப்பா

கம கமளு பனுவனளு

ஏக் மந்திரயே ரயனமோ

ரயனமல் ஏ... கமந்த்

வெல்லாயன் வெல்லம் பாளன்

வெரிகடித்தோ

உசட சாயடை உன்னும் பாணன்

கட்டாங்கரியோ

என்வாறு அமைகிறது (Shanmugam, 2018)

ஒவ்வொரு தெய்வத்துக்கும் தனித்தனிப் பாடல்கள் இருக்கின்றன. இப்பாடல்கள் தெய்வத்தின் குணவியல்களையும் வரலாற்றையும் சொல்கின்றன. மிக ஆவேசமாக வெளிப்பட்டு தானாக ஈட்டியை எடுத்து நிலத்தில் குத்தி குத்தி ஆடுவதும் நோயாளிகள், மனக்குறை உள்ளவர்களுக்கு ஈட்டியைக் கையில் கொடுத்தும் உம்பில் தடவியும் ஆற்றுப்படுத்தும் மடையில் உள்ள பூக்களை விசி விளையாடும் தெய்வம் நெடுத்தன். இத் தெய்வத்திற்கான பாடல் இவ்வாறமைகிறது என்கிறார் சண்முகம்.

குறுனாகல இல்லா இன்னா இன்னன்னொ

திம்புலாகல மறா மாப்பசுனே

சிலந்தியாவளே ஆங்கிலோ

ஏ... குறுநா மண்டானா

இப்பாடலில் வரும் 'குருநாகல’, ‘திம்புலாகல’ என்பன ஊர்களின் பெயர்கள். இவை தெய்வத்தினது அதன்படி மூதாதையரினது (இன - குடி) வரலாற்றுப் பதிவாகவும் அமைகிறது.

முன்னர் குறிப்பிட்டபடி வேடர் சமூக வழிபாட்டில் பூக்கள் முக்கிய பொருளாகின்றது. பூக்களில் தெய்வம் குடியிருப்பதாக நம்புகின்றனர். இவ்வாறு குறித்த மரங்களில் தெய்வம் குடியிருப்பதாகவும் நம்பூகின்றனர். பூ எவ்வளவு அதிகமாகக் குவிக்கப்படுகிறதோ அந்தளவுக்கு தெய்வம் மகிழ்வடையும் (Shanmugam, 2018; Velayutham, 2019). இன்றும் பூசைக்கு காடுகளிலிருந்து லாக்கடைப் பூ, காத்திகைப்பூ, பட்டிப் பூ, ஒல்லிப்பூ, ஒட்டிப்பூ, நேரம்பூ, காட்டுக் குறிஞ்சிப் பூ எனப் பல பூக்கலைக் கொணர்ந்து பூசைக்குப் படைக்கின்றனர். சடங்குச் சூழலை அலங்கரிக்கின்றனர். (Shanmugam, 2018).

திராவிட அல்லது தமிழ் மரபில், வழிபாடுகளில் ‘பூ’ முக்கியமானது 'பூ + செய்’ என்பதன் கூட்டாக 'பூசை' என்பதும் இப்பூசையை நடாத்துபவர் 'பூசாரி' எனவும் பெயர் பெற்றமை இதன் அடிப்படையிலேயே. (Baktavatchala Bharathi, 2017, p. 04) என்பதான கருத்தும் இங்கு கவனிக்கத் தக்கது. கிழக்கிலங்கை வேடர்கள் பண்டைய தமிழகத்தின் நீட்சியில் வருபவர்கள் திராவிட மரபிற்குரியவர்கள் எனும் பக்தவத்சல பாரதி (2019) அவர்களது கருத்து மேற்படி கருத்தை வலுப்படுத்துகிறது.

வேடுவர் வழிபாட்டுச் சடங்குகளில் பொதுவாக அவதானித்தக்க விடயங்களுள் ஒன்று, வழிபாட்டுப் பந்தல்கள் மட்டுமன்றி தமது குடியிருப்பு பகுதியில் ஓரிடத்தில் நடப்பட்டிருக்கும் ஈட்டியை ஒத்த வடிவுடைய கூரிய இரும்பிலான வழிபாட்டுப் பொருளாகும். இன்று வயிரவர் (காவல் தெய்வம்) தெய்வக் குறியீட்டுப் பொருளாகவும் வைதீக சமய வழிபாடுகளில் சூலாயுதமாகவும் மாற்றம் பெற்றிருப்பது இதுவே என்பதும் ஆய்வாளர் கருத்து. வாகரைப் பிரதேச வேடர்களிடம் சேனை வயிரவர் வழிபாடு காணப்பட்டதாக வேடர்குலத் தலைவர் வேலாயுதம் (2019) சொல்கிறார். மேலு கிழக்கிலங்கைத் தமிழ்க் கிராமங்களில் வீட்டுக்கு வீடு சூலாயுதத்தை ஒத்த பொருளை பந்தலில் வைத்து வயிரவர் சடங்கு நடைபெறுவது இன்றும் வழக்கிலுள்ளது. வருடம் ஒருமுறை நடைபெறும் 
இச்சடங்கில் காய்கறிகள், கிழங்குகள், தானியங்கள் என்பவைகளோடு பிரதானமான படையல்களாக கஞ்சா ரொட்டியும், கள்ளு, சாராயமும் படைக்கப்படுகின்றன.

தொழில் கருவி ஒன்று தெய்வ நிலையாக்கம் பெற்றதன் குறியீடாக இதை ஆய்வாளர்கள் (Baktavatchala Bharathi, 2017) கருதுகின்றனர். மேலும் பண்டைத் தமிழ்ச் சமூகம் தாய்வழிச் சமூகம்; இச் சமூகத்தில் பெண்ணே முதல் விவசாயி: இவ்விவசாயி பயன்படுத்திய முதற் கருவி ‘தோண்டு கழி' : இதன் மருவிய வடிவமே சூலாயுதம், இன்று அது பெண் தெய்வாங்களது கைகளிலேயே அதிகமாக காணப்படுகிறது (Baktavatchala Bharathi, 2017: p.60 - 70) எனும் கருத்தையும் இங்கு தொடர்புபடுத்த வேண்டியிருக்கிறது. கிழக்கிலங்கைத் தமிழ்ச் சமூகங்கள் தாய்வழிச் சமூக நடைமுறைகளை இன்றும் பயின்று வருவதும் இங்கு குறிப்பிடுதற்குரியது.

கிழக்கிலங்கை வேடர்களது வழிபாட்டின்வழி அடையாளப்படுத்தத்தக்க மற்றுமொரு தொல்சமயக்கூறு முருகன் கோயில் திருவிழாக்களைச் சார்ந்திருக்கினறது. இலங்கையில் இலங்கையில் பிரசித்தி பெற்ற முருகன் வழிபாட்டுத் தலமாக இருக்கும் ‘கதிர்காமம்’ தவிர்த்து அடுத்த நிலையில் கவனிக்கத்தக்க முருகன் சடங்கு வழிபாடுத் திருத்தலங்களாக அமைபவை கிழக்கிலங்கையிலே இருக்கின்றன. உகந்தை மலை, வெருகல், மண்டூர், தாந்தாமலை முதலிய தலங்கள் இவ்வகையில் கவனிப்பிற்குரியவை. தமிழ்ச் சமூகங்களது செல்வாக்கு மிகுதியாக இருப்பதன் பின்னணியில் இத்தலங்களின் மரபார்ந்த சடங்கு வழிபாட்டு மரபுகளில் மாற்றங்கள் நிகழ்ந்திருக்கின்றன. வைதீக சமய வழிபாடுகளின் செல்வாக்குகளுக்கு உட்பட்ட நிலையிலும் அவைகளிலிருந்து சற்று மாறுபட்ட சடங்கு வழிபாடுகளையே இவ்வாலயங்கள் பின்பற்றி வருகின்றன. கதிர்காமத்து வழிபாடு போன்று ‘கப்புகன்’ என்பவரே பூசைகளை வழி நடத்துகின்றார். கதிர்காமத்து உற்சவ காலத்திலேயே இவ்வாலயங்களிலும் வழிபாடுகள் நடைபெறுகின்றன.

இவ்வாலயத்திற்கான சடங்கு விழா தொடங்கும் குறித்த நாளில் அல்லது குறித்த ஒரு நாள் சடங்கில் வேடர் சமூகத்திடமிருந்து தேனும், இறைச்சியும் (மான்) கொண்டு படைக்கும் மரபு இன்னமும் வழக்கிலிருந்து வருகின்றது (Guhanathann 2020; Kedeeswaran, 2020). கோயில் மூர்த்திக்கு படைக்கும் மரபு விலக்கப்பட்டிருப்பினும் இப்படையல் தொடர்கிறது. மட்டக்களப்பில் கொக்கட்டிச்சோலை தான்தோன்றீஸ்வரர் ஆலய வழிபாடுகளிலும் இம்மரபைக் காணமுடிகின்றது. (Guhanathann 2020). மொத்தத்தில் வேடர்களது வழிபாட்டிடங்களும் வழிபாட்டு முறைமைகளும் பெருஞ் சமூகங்களது செல்வாக்குக்கு உட்பட்டுப் போன வரலாற்றையே இந்நடைமுறைகள் தெரிவிக்கின்றன. பெருஞ் சமூகங்களுடன் கலப்புற்றுப் போன வேடர்களது சமூக வாழ்வையுமே இது காட்டுகிறது. மேலாக இறைச்சி முதலானவற்றை படைத்து வழிபடும் தொல்சமய மரபின் தொடர்ச்சியை வெளிப்படுத்துபவையாகவும் இவை அமைகின்றன.

\section{முடிவுரை}

கிழக்கிலங்கையில் வேடர் சமூகம் பரவலாக வாழ்ந்து வருகின்றது. மரபார்ந்த இனக்குழு வாழ்வைத் தொடர்வதில் மாற்றங்களையம் சிக்கல்களையும் எதிர்கொண்டு வந்திருக்கின்றது. எனினும் இனக்குழு வாழ்வின் அடிப்படைகளை முற்றாக இழக்கவில்லை. வழிபாட்டுச் சடங்குகள் இதனைத் தெளிவுபடுத்துகின்றன. இறந்தோரை வழிபடல், இறைச்சி முதலானவற்றை படையல்களாக்கல், ஆவி பற்றிய நம்பிக்கை, அவை (ஆவி) மானிட உடல்களுள் குடிபுகுந்து இயக்குமென்ற நம்பிக்கை; தம்மை மீறிய சக்தி ஒன்றுண்டு என்ற நம்பிக்கையும் அச்சக்தியை தம்மால் வயப்படுத்த முடியும் என்ற நம்பிக்கையும் எனத் தொல் சமய அடிப்படைகள் வேடர்களிடம் காணப்படுகின்றன. இவை அவர்களது வழிபாட்டுச் சடங்குகளில் வெளிப்படுகின்றன. இச்சடங்கு வழிபாடுகளில் வேடர்களை அடையாளப்படுத்தும் இனக்குழு வாழ்வின் அடிப்படைகள் பலவும் வெளிப்படுகின்றன. தொழில், உணவு, இயற்கையுடன் இணைந்த கூட்டு வாழ்வு, தனித்துவமான மொழிவழக்கு முதலான பல விடயங்களை சடங்குகள் வழி அடையாளங் காணமுடிகிறது. சடங்குகள் வழி இவற்றை நிலைப்படுத்த முடியும் என்பதும் தெளிவாகின்றது. 


\section{References}

Baktavatchala Bharathi, (2017) Cultural Dialogue, Putha nanndham adayalayam, Sri Lanka.

Sivasubramaniyan, A., (2017) Sangam Literature and Archaic religion, New Research Journal, 09, 184-204.

Sivasubramaniyan, A., (2018) Disintegration and religious formation of ancient Tamil Nadu, New Research Journal, 09, 16-34.

Thangarasa, U.V., (1994) An article on the impact of ethnic identity on eastern coastal veddas .'Article in the Collection of Reading sri Lankan society and culture, Editor-Sanathan, T., Colombo: Colombo Institute of Socio-Cultural Studies, Sri Lanka.

\section{Information provider}

Shanmugam, G., Age 64, Vantharu Moolai, Dtd 18.06.2018.

Velayutham, P., Ahe 58, Kunjan kulam, Dtd 23.12.2019.

Guhanathan, M., Age 32, Kokkaddicholai, Dtd 20.01.2020.

Kedeeswaran, M., Age 27, Munaikadu, Dtd 20.01.2020.

Funding: NIL

Acknowledgement: NIL

Conflict of Interest: NIL

About the License:

\section{(C)(1)}

Attribution 4.0 International (CC BY 4.0)
(C) The author 2021. The text of this article is licensed under a Creative Commons Attribution 4.0 International License 